\title{
DR. GEO II: Adding Interactivity Planes in Interactive Dynamic Geometry
}

\author{
Hilaire Fernandes, Stéphane Ducasse, Thibault Carron \\ University of Savoy - LISTIC, SYSCOM labs \\ Annecy, Le Bourget du Lac, France \\ \{hilaire.fernandes,stephane.ducasse,thibault.carron\}@univ-savoie.fr \\ Accepted to International Conference on Creating, Connecting and Collaborating through Computing (C5 2007)
}

\begin{abstract}
Interactive geometry environments support the creation and exploitation of interactive geometric sketches. However, such environments are often driven in a rigid manner, following a well specified construction path. This rigidity is not always compatible with: $i$. the internal cognitive representation of the learner about the geometric domain and ii. the way a geometric sketch is used in a paper-pen environment. This rigidity is therefore a source of internal tension for the learner and it can reduce the pedagogical added value of the interactive geometry environments. We think additional interactive planes to manipulate a geometric sketch differently can help the learner. We have developed DR. GEO II, an interactive geometry framework that is able to receive additional interactive planes such as a free sketching and a command-based one. We have experimented it in a junior high school class and we report here our first results.
\end{abstract}

\section{Introduction}

Interactive geometry environments, such as Cabri Géomètre [4] which appeared in the '80s, give the possibility to define a geometric construction according to its internal properties. For example, a circumcenter is defined as the intersection point of the vertices of the triangle. Moreover, such environments enable the learner to construct the geometry sketch interactively and move some of its parts dynamically according to its internal properties. With our example, moving one triangle side will recompute the circumcenter dynamically. Interactive geometry software is, in fact, a constraint system specialized for the geometric domain. Other constraint systems such as Sketchpad [15] and ThingLab [5] proposed a more general approach to handling geometric, mechanical or electrical constraints. Because of their specialization for the geometric domain, interactive geometry environments are better suited to a pedagogical use, in particular the user interface and didactic aspects are better defined.

However, interactive geometry environments - like every instrument - impose one specific way to represent, interact and manipulate a domain. The learner mediated activity is more or less structured depending on the model imposed by the software rather than on the learner's internal representation of the domain studied. This results in cognitive tensions between these two representations and hampers the learning activity.

To lower these tensions, it is interesting to make the software model closer to the learner's internal representation instead of imposing the software model on the learner. The learner's internal representation is often modeled after years of learning based on empirical activities with non-virtual tools such as paper, pens, eraser, ruler, compasses, wire, etc. When the learner is acting within a complete virtual environment such as interactive geometry software, there is a big qualitative and conceptual gap where the empirical way of approaching things is not easy. Reducing this gap can make interactive geometric environments more efficient pedagogically.

Our research hypothesis is therefore to propose different modes of action to the learner when $\mathrm{s} / \mathrm{he}$ manipulates a geometric sketch. According to the problem-domain studied, the learner uses a specific mode, that we call interactive plane. In particular, we believe that repositioning the empirical approach in the environment and activities is important. To test our hypothesis the first author developed DR. GEO II an interactive and extendable geometry framework with interactivity planes $[7]^{1}$. We have experimented this system in a junior high school, in year 4, in Mont de Marsan, France. Our article is structured as follows: the first section lists the problems linked to instrumented activities. We then introduce the notion of interaction planes. Next we describe the experimentation we conducted and conclude with perspec-

\footnotetext{
${ }^{1}$ DR. GEO II is inspired by the DR. GEO software, free interactive geometry software developed for Linux by the same author, http:// www. of set.org/drgeo
} 
tives and evolutions.

\section{Instrumented Activities}

When a teacher prepares an activity with a geometric environment, s/he introduces a tool, the software, that is an auxiliary between the learner and her/his activity[10]. According to the Vygotsky theory of activity, the learner always operates on an activity with an intermediate auxiliary, a tool. This auxiliary imposes a representation, more or less internalized by the learner. This representation depends on the learner and the domain[3].

For Béguin [6], the tool is first an artifact instrumented in a unique manner by each learner. Two different persons, according to their personal experiences, can use differently a given tool: the tool is instrumented differently. The way an artifact is instrumented depends on the learner's knowledge, the activity and the environment. In his study, Béguin compares the use of a paper-pen or computer based environment in the context of an electric diagram designer. The comparison illustrates the rigidity of the computerized environment that makes it difficult for the designer to instrument the computer environment (artifact) just as s/he was able to do with a simple paper-pen environment (artifact). The artifact for him is the tool as it is designed (for example a wrench), the instrumentalisation of the artifact is the way it is used (using a wrench as a hammer). The instrumentalisation is then an artifact transformation process, according to the activity and the social schemes of the operator. However, he stresses that instrumentalisation of a computerized artifact is not impossible, although it is more difficult.

The instrumentalisation of computerized artifacts can be difficult when the artifacts are not compatible with the learner's socio-cultural schemes. Soury-Lavergne [14] in her experimentation with distance tutoring using Cabri, reports the students' difficulties to construct a segment median. Indeed in this example, the computerized artifacts to use for this construction are "construct a circle" and "construct a segment". The learner cannot find the compasses artifact that s/he was used to in a paper-pen environment and $\mathrm{s} / \mathrm{he}$ had difficulties in the instrumentalising of the construct circle software artifact to determine two points of the median. This difficulty was also reported by the teacher we worked with during our experimentation (see Section 5). Here the students' difficulty is not only related to their knowledge - they know how to construct a median but they have not yet conceptually linked it to the construction of two circles with identical radiuses. The difficulty is more related to the operative mode of the software artifacts.

Our problem is therefore centered around the artifacts and their instrumentalisations by the learners. To be instrumented, the artifacts have to be in the scope of the learners' socio-cultural schemes. Such artifacts should be used in the same activity, providing different points of view on it.

\section{Interaction Planes}

We call interactivity planes a collection of user interfaces at the disposal of the learners to operate in different manners on the same pedagogical activity. In the specific context of interactive geometry, we propose an environment, DR. GEO II, where such planes can be easily added and developed. These planes propose a coherent multi-faceted world in geometry activities. The learners can switch from one plane to the other during the same activity. In the same way, it is related to the multi-modality user interface paradigm [13].

To offer this flexibility, we have designed our interactive geometry framework DR. GEO II in the dynamic Squeak/Smalltalk environment $[9,11]$. In Squeak/Smalltalk the different constitutive parts can easily communicate with each other. DR. GEO II is designed to be smoothly integrated and to become a communicative part of this system, and to benefit from the whole dynamic it offers.

\section{Examples of Interactivity Planes}

DR. GEO II offers three interactivity planes: a logical construction, a free-hand sketching and a command one. A fourth one based on hand-strokes recognition is in early development stage and it is not discussed there.

\subsection{Logical Construction Plane}

Description. It is the classic interactivity plane of the interactive geometry software, it enables the user to construct logically different objects: point, line, value; construction with geometric transformations, relations between different objects (belong to, middle, dependency of a point to a value).

To construct a segment or a straight line, the user creates two points directly on the screen, then when pointing at these two points, s/he defines the segment or straight line.

Hypothesis. This plane enables the learner to construct quickly a geometric construction according to its description. The hypothesis here is that the learner can evaluate directly if her/his hypotheses about a given construction are correct. The use of this plane is relatively intuitive because the user does not manipulate the formal symbols directly, but only their graphical representation. It results, however, in a logical geometric construction.

Example. DR. GEO II implementation contains the main tools expected for logical geometric constructions: points, 


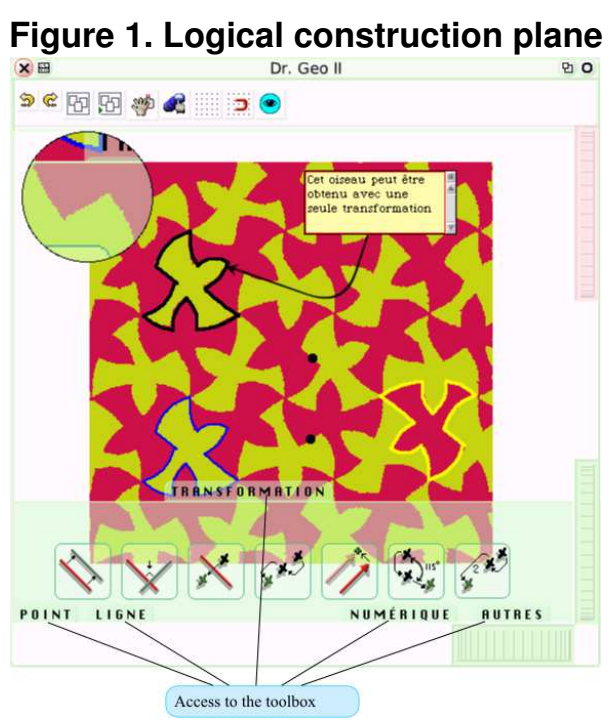

lines, geometric transformation and some other functions to facilitate such manipulation as group command, magnetic grid, an undo/redo stack. Some features such as locus and macro-construction were not implemented as they were not necessary for our study.

The tools are arranged in translucent green flaps, at the bottom of the view. A flap is automatically opened when the mouse moves over it. About twenty tools are located in the flaps POINT, LINE, TRANSFORMATION, NUMERIC and OTHERS. Rotating wheels are used to move and zoom the figure. Figure 1 shows DR. GEO II with the transformation flap opened.

Comments. The logical construction plane integrates the classical functions of dynamic geometry software: construction of point, segment, straight line, radius, circle, arc, vector. Note that vectors can be manipulated as they should be: A vector's graphical representation can be moved independently from its extremities. In a number of geometry environments, such is not the case, which is pedagogically inconsistent. The vectors are used to define translation and oriented angle. Points (free, on a line, intersection) can be created on the fly when constructing a line object.

\subsection{Free-hand sketching plane}

A second interactivity plane we use in DR. GEO II is a free hand sketching interactivity plane - or drawing plane.

Description. To commute to this plane, the user just drops a paint icon in a DR. GEO II window. From there, the user can draw freely independently of the logical geometric construction plane. The resulting sketch can be moved into the geometric window or be thrown away. It is possible to draw different disjointed elements manipulable separately.

Hypothesis. Our hypothesis with such a plane is that it offers more freedom to explore a geometric drawing. In particular this plane is close to the pen-paper habits of the learner. In this plane, the pure logical dimension of the environment is set aside for a more experimental approach such as students can have on a draft document: freedom to make annotation, to draw point, line, color in an unrestricted manner. This plane can be activated at any time and it supplants thus, for a moment, the geometric sketch the student was elaborating.

Example. In figure 2, on the left, the user drops the Palette icon in a geometric construction. The overall geometric drawing is set in a pause mode. A new toolbox appears - partial view on the right in figure 2. Here we can see the free line tools used to determine a symmetry center. To exit from the drawing plane, the user clicks on the button FINI (Achieved). The resulting drawing is then integrated into the geometric drawing where it can be moved, edited or deleted.

Figure 2. Use of the drawing plane
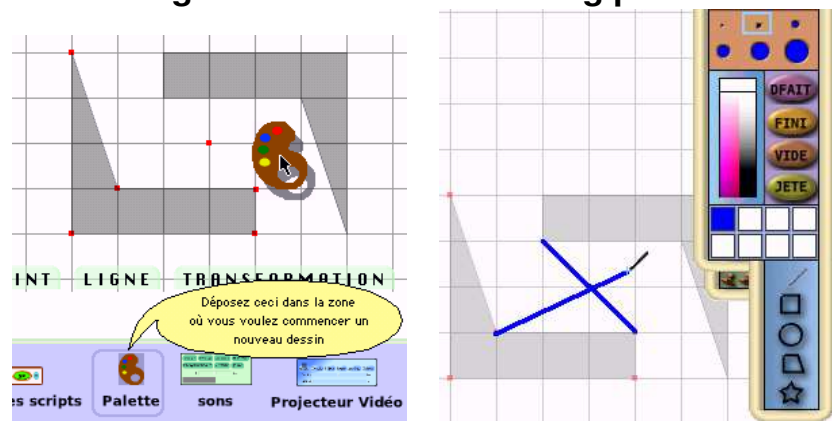

Figure 3 presents an overall view of the free-hand drawing interactivity plane. The student has used drawing to verify and to determine the symmetry center of two fish-bird tiles.

Comments. As defined presently, the drawing tools are simple and are not specialized for geometric drawing. However these drawing tools help to explore hypotheses without the constraints of the logical geometric construction tool this was the effect we were looking for. As a counterpart, the fact that the tools are free may offer too much freedom to the learner.

This interactivity plane is a Squeak multimedia component integrated in DR. GEO II. As explained previously, our framework was designed to be compatible with the existing media of the Squeak environment. 


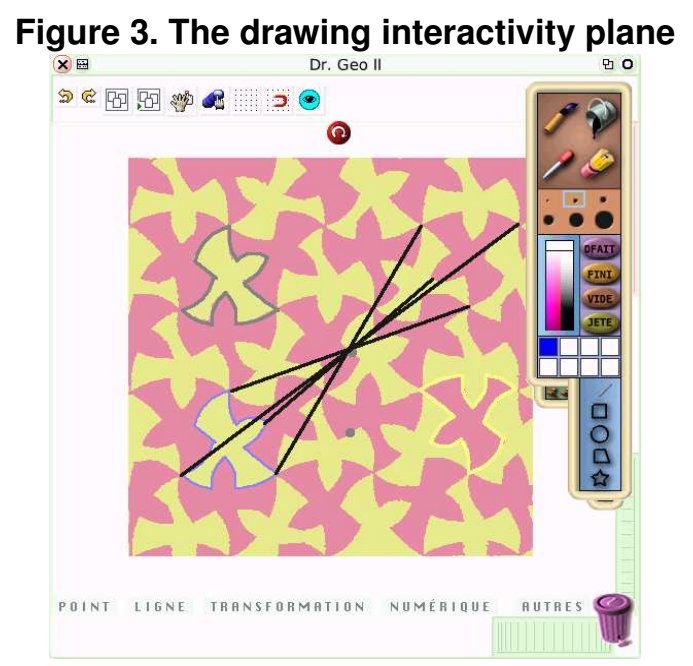

\subsection{Command interactivity plane}

The third interactivity plane we have explored is object manipulation through commands.

Description. Using graphical and pluggable commands provided by the Etoys environment $[2,8]$, the learner can script objects. With Etoys, the user can design script with graphical commands acting on user interface components. The command categories are various: move, transformation, aspect, simple calculus. For the time being, these commands can be applied to point and numerical objects.

Hypothesis. Our hypothesis is that such a plane helps the learner to understand the conceptual relation existing between the euclidean aspect of a geometric figure, its numerical dimension - coordinates, functions - and the numerical relations linking its geometrical objects - for example numerical relations with the coordinates of the middle of two points. This interactivity plane provides the opportunity to manipulate objects with numerical commands, thanks to an artifact offering a comfortable user interface, without complex command manipulation.

Example. Although this interactivity plane can be activated for any geometric object, for now it is only interesting for point and numerical objects. This plane looks like a command panel to modify object attributes. With a point object, the user can modify its coordinates. Figure 4 shows the command panel of a point $\mathrm{A}$. Its coordinates $-\mathrm{Xw}$ and Yw in the panel - can be modified with the input of a new value or with the small green up and down arrows. The position of point $\mathrm{A}$ and its dependencies are adjusted accordingly.

\section{Figure 4. Command panel of the point $A$ to modify its coordinates.}

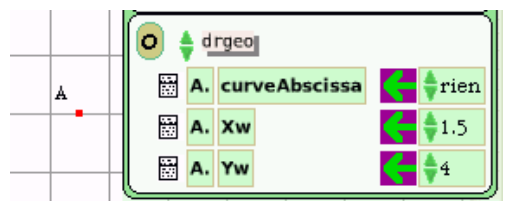

In relation with our hypothesis, the interesting aspect is the ability to link different objects numerically thanks to the numerical commands of the panel. In the following paragraph, we describe a connection between the point A coordinates and a joystick widget.

Figure 5 shows on the top the creation of a command script to modify the abscissa of point A. A script is simply a series of Etoys commands. It results in a script panel - pictured on the bottom - where commands from various objects are stacked. The picture on the bottom shows such a script panel with one command coming from a joystick object. When it's finished, this panel script lets the user manipulate with the joystick the horizontal position of point A. This simple example shows the possibility to link different objects, even if some of these objects are not part of our geometric framework, here the joystick widget.

Figure 5. Stack of commands

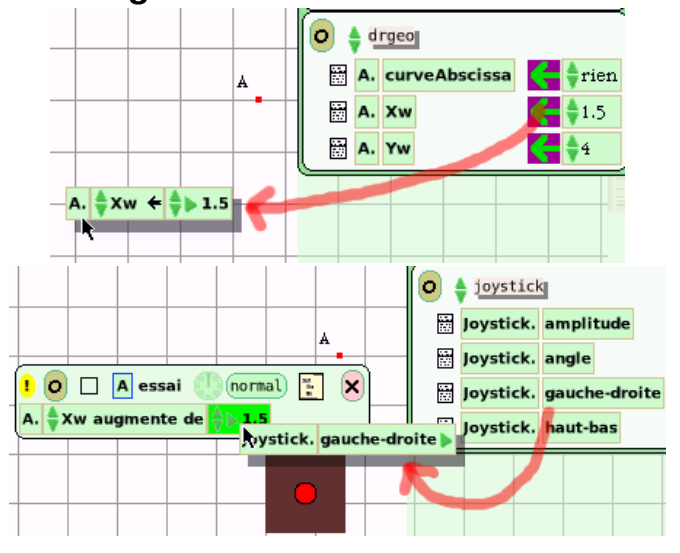

In figure 6, a set of graphical commands and a joystick are used to modify the position of point $\mathrm{A}$ according to the $\mathrm{x}$ and $\mathrm{y}$ axis. A divider may be added to the script panel to reduce the amplitude of the point movement.

Comments. An interesting feature of Etoys is the possibility to link different objects which at first were not meant to be used together. For example, using a virtual joystick to control a geometric sketch object's position, dropping pic- 


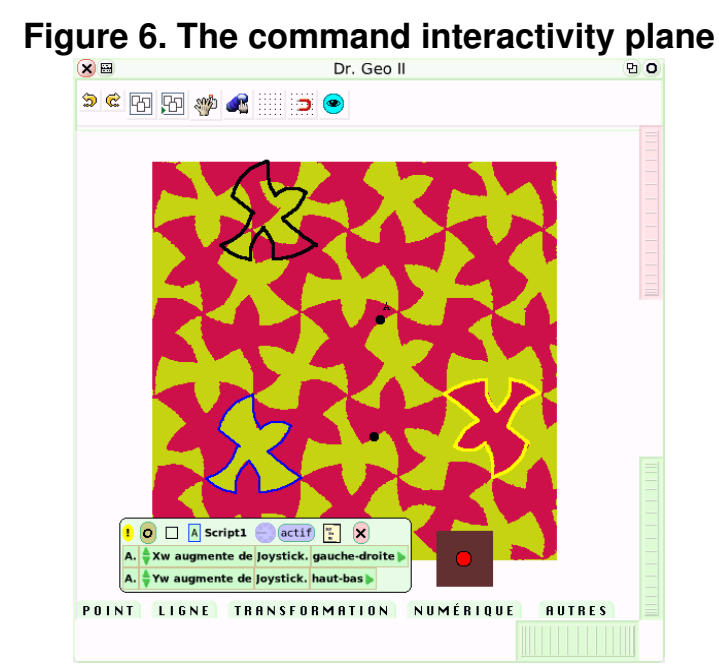

tures into the geometric sketch.

It is possible to elaborate various uses of this interactivity plane: cascading interactivity with different points and widgets, commands compelling a point to remain in a given area, etc. When linking dynamic geometry, numerical consideration and graphical programing, this interactivity plane offers an open area for original pedagogical use.

\section{Experimentation}

Since 2001 the Landes district in France has been conducting a "one high school student, one notebook" operation in which every teacher and student receive, for the school year, a notebook computer they can take home [1].

We set up a user experimentation for the interactivity planes. The experiment was conducted in a 25 student junior high school class of pupils aged 14. Each student was using her/his own notebook. Most of the experiment took place in the classroom, a tiny part was homework.

With the mathematics teacher we set up an activity related to central symmetry and translation transformation. The activity was designed to use the free-hand drawing and command interactivity planes. The experiment was organized into three 50 min periods: one period for preparation and two for the activity itself.

The activity was using a tessellation with geometric transformations - central symmetry and translation. Also in this artwork, the composition of two central symmetries can be linked to a translation transformation. The main aspect of the activity was therefore around central symmetry and the composition of two central symmetries resulting in a translation.

\subsection{Period 1: Preparatory phase}

This period was necessary to prepare the students for the DR. GEO II environment and its interactivity planes. The students received a work sheet related to triangle and geometric transformations. With this document they experimented the use of the three interactivity planes we presented them with in the previous sections. This preparatory period was very important in order to get them into the right conditions for the next periods of the experiment.

\subsection{Period 2: Activity}

For periods 2 and 3, the students received another work sheet and a previously prepared DR. GEO II geometric figure. This figure was a tessellation and a composite geometric object - a group of segments and arcs - outlining a selected basic fish-bird tile.

The students searched for and determined - with the help of the free-hand drawing interactivity plane - the symmetry center of a configuration composed of two basic tiles. In order to do so, they had to conjecture on possible symmetric tiles. And just as they would have done in a paper-pen configuration, they had to determine this by drawing the position of a symmetric center. Some of them succeeded in doing so, others failed, but all of them did so in an active free approach, freed from the constraints of the geometry environment.

Once the center was determined, they constructed, with the logical interactivity plane, the transformation of our selected basic tile. The point of the drawing interactivity plane is not to overload the research phase with too much formalism.

\section{Figure 7. Student work in period 2}
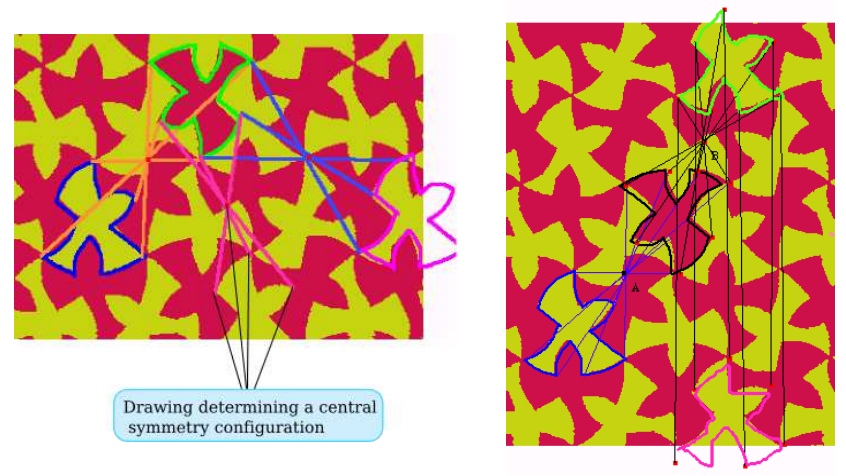

Figure 7 shows two students' production. On the left, the student determined three configurations of central symmetry: using groups of blue, orange and magenta lines. In two of the three configurations, the student constructed the transformed tiles: the magenta and green tiles. The screenshot on the right shows a reverse use of the logical and 
drawing interactivity plane: the logical interactivity plane was used first to determine symmetry centers, and then the drawing one to draw the outline of the transformed basic tile.

The pedagogical objective of this period was the construction of two successive symmetric tiles, resulting in two symmetry centers. For homework, the students were asked to construct a command controlled with a joystick to move the first symmetry center. The objective for the next period was to link it to the translation.

\subsection{Period 3: Activity}

During period 3, the student had to link the composition of the two symmetries to a translation. The drawing interactivity plane was again used to show the parallelogram configuration, related to translation transformation: creation of a segment joining two summits, duplication of this segment with the clone tool of the Palette to show the parallelogram configuration.

The left screenshot in figure 8 shows a student drawings to highlight the parallelogram configuration of the translation. In this screenshot, the brown tile (middle) is the symmetric of the blue tile (left) through point $\mathrm{A}$, the yellow tile (right) is the symmetric of the brown tile through point B. The student, when drawing the related parallelogram, has shown the translation relation between the yellow and blue tiles.

Next, with the logical interactivity plane, the student has used the vector and translation tools to construct the translation of the blue tile - resulting in a black tile perfectly superposed onto the yellow one.

Next, with the command interactivity plane, the students graphically programmed the position of the two symmetry centers. These two centers were moved to determine several configurations of central symmetries and translations (right screenshot, figure 8): with the two joysticks, the students moved the centers and determined translation configurations in the artwork.

\section{Figure 8. Student work in period 3}
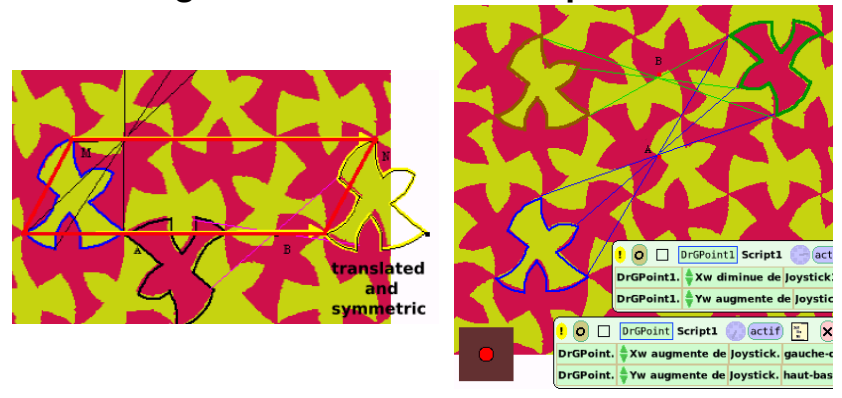

\subsection{Analysis}

We discuss the overall results, then we present some feedback from the teacher and the students.

Overall results. During such a short experiment, the adaptation phase to the software environment takes a lot of time, which can be a problem for the pedagogical activity itself. The students can be stuck if they do not master the environment, and they have to dedicate an important part of the activity time to getting used to the software.

This is why we introduced the 1 st period as a preparation one. A greater familiarity would have been useful. In our case, the students were familiar with other geometry software the teacher had introduced earlier in the school year. Therefore the students were used to different user interface paradigms to construct geometric objects.

The free-hand drawing interactivity plane was well perceived. It was useful during the research phase. Our experiment confirmed our hypothesis regarding this interactivity plane. With the logical construction plane, the students would use advanced geometric tools, with their inherent formalism. Indeed, the logical construction plane is suited to construct quickly a geometric sketch but the learner cost to internalize it is higher - see our discussion with the students.

The command interactivity plane brings non negligible cognitive difficulties to students not used to this kind of manipulation. Its use in one activity is probably not enough to have an accurate conclusion about its usefulness. Nevertheless, the teacher agreed with our hypothesis regarding its interest to bridge together different mathematics domains.

Discussion with the teacher. The activity was designed closely with the math teacher. After the experiment, we prepared a few questions to analyze how she perceived these periods. We report her answers below, then we comment on the next section.

1. Did the students have difficulty in switching from the logical interactivity plane to the drawing one?

No, they did not.

2. Did the manipulation in the drawing interactivity plane give some operative or cognitive difficulties? No major difficulties here but one annoying point. With the drawing interactivity plane, all the drawings are grouped in one layer: when a student draws several segments, all of them are grouped in the same layer and cannot be moved independently. To get independent drawings, you have to quit the drawing interactivity plane for the logical interactivity plane, then enter again a new drawing interactivity plane. It increases the number of manipulations. 
3. Do you think the drawing interactivity plane was useful to the students?

No. In fact the students are used to doing experimentation and research with interactive geometry software, therefore they are able to adjust the position of the constructed elements. Which is not possible when using the DR. GEO II drawing interactivity plane.

4. What difficulties did the students have with the command interactivity plane? Conceptual ones? Operative ones?

Conceptual difficulties are important with the command interactivity plane because it is linked to notions quite unknown to the students: function, variable, script of commands.

On the other hand, the command interactivity plane is quite easy to manipulate, the video-tutorial distributed was useful to the students.

5. Could the manipulation with commands help the students to link the geometric and numeric dimensions? Yes, even though we did not get very far in this direction, it is a very interesting and promising direction.

6. According to you what did not work very well? We did not reach clearly the objective of the activity: clearly linking the composition of two central symmetries in a translation.

7. According to you what did work very well? During the second period, not very advanced students did quite well while working in autonomy. They got interesting results.

8. If we should repeat the same experiment, what should we change?

- Use a 2 block-period. Periods 2 and 3 were separated by a long 3 day weekend. In period 3, the students had forgotten what they did during period 2. At least periods 2 and 3 should be on two consecutive days.

- Make the students more familiar with the software environment.

- Some bugs and manipulation of the software perturbed the students.

Analysis of the Experiment Results. The drawing interactivity plane as designed and used, does not create conceptual or operative difficulties. However the teacher thinks it is not very useful. Indeed most of the operation in this mode can be reproduced with the tools of the logical interactivity plane, although the level of complexity is not the same in the two planes. In the proposed activity, the drawings can be easily reproduced with the geometry tool. However, with more complex constructions, the students faces the formalism inherent to this interactivity plane. To date, DR. GEO II is the only software proposing both the drawing and logical interactivity plane.

One student reversed the use of the drawing and logical interactivity planes. It appears the student did not know about the DR. GEO II group function which facilitates the transformation of aggregated objects. So the student used the drawing plane to represent a complex transformed object. It is an interesting example of the instrumentalisation of an artifact.

Some students, usually not advanced ones, did quite well during period 2. Period 2 started with the use of the drawing interactivity plane. We can question whether this can be linked to the use of this specific plane. An interview with the students, presented below, clarifies this.

Regarding the command interactivity plane, the teacher thinks it is promising. Even if it is conceptually more complex, it's still in the students' zone of proximal development: all the newly introduced concepts are in the learning sphere of the students. Moreover, this plane, as designed in DR. GEO II, does not introduce complex manipulations. Nevertheless, the new pedagogical possibilities need to be explored. Our experimentation was not centered on this specific plane but on the concurrent uses of the three interactivity planes. To evaluate the command plane correctly, it is necessary to set up a recurrent experiment in which the teacher and students can get comfortable with this plane.

Discussion with some students. After the interview with the teacher and her analysis, we conducted an interview with selected students. These students were:

- In group A, usually students with a low score but having done quite well during period 2;

- In group B, we asked the teacher to select students usually comfortable with interactive geometry software.

The goal of this interview was to examine how the students perceived the free-hand drawing interactivity plane.

1. Did you find it difficult to use the drawing tools in the DR. GEO II geometry software?

The students did not have difficulty in using the drawing interactivity plane nor in commuting between this plane and the logical one.

2. Do you think the drawing tools were useful?

Students of group B quickly responded no. When i asked them if it would have been easier with the geometric tools, they replied yes. When I asked how they 
would have gone about it, the different steps of the constructions were not clear but they felt quite confident they could do it.

Then group A continued to argue that the drawing tools were useful for them. They felt it was easier for them to start first with the free-hand drawing tools then to go on with the logical interactivity plane. It was interesting to note that these students were not sure they should argue for the positiveness of the free-hand drawing tools, especially after students of group B argued the opposite.

This feedback is interesting, it confirms that, for students already comfortable with the formalism of the interactive geometry software they have been using during the year, the drawing interactivity plane is not perceived as useful for this activity. These students have already internalized the artifacts of the geometry software, they are able to instrument them.

However, for the other students, the drawing interactivity plane was a useful auxiliary, an environment between paperpen and the logical construction plane they still have some difficulties in mastering. Therefore when mixing these two interactivity planes we help the students' social-cultural representations to get closer to the artifacts of the logical interactivity plane.

\section{Conclusions}

Our experimentation has comforted our hypothesis on the importance of transposing the paper-pen environment to a computerized geometric environment. Students who were not very comfortable with the formalism of classic geometric software felt more capable of conducting some parts of an activity with the drawing interactivity plane. Indeed its unformal aspect gives more freedom to the students, especially in the drawing aspect of a geometric construction.

However our idea is not to replace the logical construction plane with the drawing one, but to mix these two approaches, so the students can get used to the formal approach of interactive geometry smoothly. For students already used to this formalism, they perceive it as unnecessary. Perhaps with more sophisticated tools (rulers, compasses,...) the drawing interactivity tools can be perceived differently by these students.

Our command interactivity plane proposes a programming approach to interactive geometry manipulation, its specific pedagogical use needs additional experimentation but our results already reveal that its user interface - based on the Etoys framework - is perfectly suited to the students.

Another important point, not discussed here, is the necessity of being able to observe accurately what is done by each student. We will specify, as future work, the concept of "pedagogical interactive textbook" from which we can observe and save some specific actions carried out by the students. These records can be used later by the teacher or the student to replay and analyse specific moments of the pedagogical session.

In the future we will attempt to enhance the drawing interactivity plane with artifacts closer to the geometric domain: ruler, compasses, setsquare, protractor. Also it will be useful to transform objects from the drawing plane to the logical one: a segment drawn in the drawing plane could be interpreted and transformed as a logical segment of the logical plane, as is the case in the Cinderella software[12].

\section{References}

[1] C. G. des Landes. http://www . landesinteractives. net, 2002.

[2] B. Allen-Conn and K. Rose. Powerful Ideas in the Classroom. Viewpoints Research Institute, Inc., 2003.

[3] M. BAKER, E. de VRIES, K. Lund, and M. QUiGNARD. Computer-mediated epistemic interactions for coconstructing scientific notions: Lessons learned from a fiveyear research programme. In Proceedings of EuroCSCL 2001, pages 89-96, March 2001.

[4] F. Bellemain. Un cahier de brouillon informatisé pour la résolution de problèmes de géométrie plane. Petit $x$, (16):35-48, 1988.

[5] A. Borning. Thinglab - a constraint - oriented simulation laboratory. Technical report, Xerox - Palo Alto research center, July 1979.

[6] P. BÉGuin and P. RABARdel. Designing for instrumentmediated activity. Scandinavian Journal of Information Systems, (12):173-189, 2000.

[7] H. FERnANDES. Dr.GeoII, an interactive geometric framework in squeak/smalltalk. http://www. squeaksource.com/DrGeoII.html, 2006.

[8] M. Gaelli, O. Nierstrasz, and S. StinckWich. Idioms for Composing Games with EToys. In Proceedings of C5 2006, Jan. 2006. To appear.

[9] A. Goldberg and D. Robson. Smalltalk 80: the Language and its Implementation. Addison Wesley, May 1983.

[10] I. IVIC. Lev S. Vygotsky. Prospects:the quarterly review of comparative education, XXIV(3-4):471-485, 1994.

[11] A. C. KAY. The early history of Smalltalk. In ACM SIGPLAN Notices, volume 28, pages 69-95. ACM Press, Mar. 1993.

[12] D. Materlik. Using sketch recognition to enhance the human-computer interface of geometry software. Master's thesis, Freie Universität Berlin, Institut für Informatik, 2003.

[13] J. PrEECE. Human-Computer Interaction. Addison-Wesley, 1994.

[14] S. Soury-Lavergne. Connaissances et mise en œuvre d'un micromonde dans les interactions de préceptorat distant. STE, 8(3-4):321-345, 2001.

[15] I. SuTHERLAND. Sketchpad, a man-machine graphical communication system. In AFIPS Spring Joint Computer Conference, pages 329-346, May 1963. 